\title{
Kernos
}

Revue internationale et pluridisciplinaire de religion grecque antique

$13 \mid 2000$

Varia

\section{Das Hypogäum auf der Agora von Poseidonia: ein Kultort der Tritopatores?}

\section{Mario Rausch}

\section{OpenEdition \\ Journals}

\section{Édition électronique}

URL : http://journals.openedition.org/kernos/1297

DOI : 10.4000/kernos. 1297

ISSN : 2034-7871

\section{Éditeur}

Centre international d'étude de la religion grecque antique

\section{Édition imprimée}

Date de publication : 1 janvier 2000

ISSN : 0776-3824

\section{Référence électronique}

Mario Rausch, « Das Hypogäum auf der Agora von Poseidonia: ein Kultort der Tritopatores? », Kernos [En ligne], 13 | 2000, mis en ligne le 21 avril 2011, consulté le 01 mai 2019. URL : http:// journals.openedition.org/kernos/1297 ; DOI : 10.4000/kernos.1297 
Kernos, 13 (2000), p. 107-116.

\section{Das Hypogäum auf der Agora von Poseidonia: ein Kultort der Tritopatores?}

Seit der Publikation betreffend einen auf der Agora von Poseidonia gefundenen unterirdischen Kammerbau wurden aufgrund seiner architektonischen Form, seiner Lage und der Beifunde höchst unterschiedliche Deutungsvorschläge vertreten ${ }^{1}$. So wurde die ursprünglich von einem Hügel bedeckte Anlage als Heiligtum einer chthonischen Fruchtbarkeitsgöttin, als Kultort einer bzw. mehrerer Nymphen, als Heiligtum eines mythischen Heros oder des heroisierten Gründers von Sybaris interpretiert ${ }^{2}$.

Hier soll gezeigt werden, daß die Errichtung dieses Kultbaus am Westrand der Agora im Rahmen einer im späten 6. Jh. v. Chr. durchgeführten Definition der Grenzen dieses öffentlich nutzbaren Bereichs erfolgte. Darüber hinaus soll der hier vollzogene Kult mit jenen Zeremonien zu Ehren der Tritopatores verglichen werden, die in einem kürzlich veröffentlichten Sakralgesetz der westsizilischen Stadt Selinunt beschrieben werden. Aufgrund der dabei feststellbaren Übereinstimmungen soll für das Hypogäum in Poseidonia eine Deutung als Monument der Tritopatores vorgeschlagen und deren Konsequenzen diskutiert werden. Zunächst gilt es jedoch, nochmals die gesicherten Erkenntnisse über dieses Monument zusammenzufassen.

1 Zu den von P.C. Sestieri, Il Sacello-Heroon, in Boll. d'Arte, 40 (1955), S. 53-64, publizierten Grabungsergebnissen und zu den Funden innerhalb der Kammer vor allem U. Kron, Zum Hypogäum von Paestum, in Jdr, 86 (1971), S. 117-148. Zum archäologischen Befund E. Greco, in D. Theodorescu, E. Greco, Poseidonia-Paestum II, L'agora, Rom, 1983 (Coll. de l'École française de Rome, 42), S. 28-33, zum Inventar der Grabkammer S. 78; die im Peribolos dieses Monuments gemachten Kleinfunde sind in einem Katalogteil, S. 139-145, Nrn. 259-325 aufgelistet.

2 Heiligtum einer auf einen einheimischen Fruchtbarkeitskult zurückreichenden chthonischen Gottheit Hera-Demeter: Sestrenr, ant. cit. (n. 1), S. 55f., 61; Nymphenheilig-

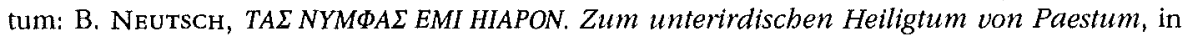
Abb. Heidelberg (1957), 2, S. 18ff,; mythischer Heros: Greco, op. cit. (n. 1), S. 78f.; Gründer von Sybaris: P. Zancani Montuono, Il Poseidonion di Posidonia, in ArchstorCalabria, 23 (1954), S. 183ff. $\mathrm{Zu}$ den unterschiedlichen Deutungsvorschlägen, Kron, art. cit. (n. 1), S. 124ff, sowie allgemein J. Pedley, Paestum. Greeks and Romans in Soutbern Italy, London, 1990, S. $37 f$. 


\section{Die Lage des Monuments auf der Agora von Poseidonia}

Die topographische und archäologische Untersuchung des Stadtgebietes von Poseidonia hat gezeigt, daß ein großes, unbebautes Areal bereits bei der Aufteilung des Siedlungsgebietes im frühen 6. Jh. frei gehalten wurde und wohl von Anfang an als öffentlich nutzbare Fläche, als Agora, diente ${ }^{3}$. Diese umfaßte eine zwischen den beiden großen Stadtheiligtümern im Norden und Süden gelegene leichte Senke, die im Westen unmittelbar an das Wohngebiet angrenzte ${ }^{4}$. Das hier diskutierte Monument wurde im nördlichen Bereich der Agora (ca. $70 \mathrm{~m}$ südlich des Stadtheiligtums der Athena) und gleichzeitig an ihrem westlichen Rand errichtet und nach Osten, auf die Freifläche hin, orientiert.

\section{Die Form des Gesamtmonuments, das Aussehen und der Inhalt der unterirdischen Kammer}

Das hier angelegte Monument bestand ursprünglich aus einer von Erde überdeckten kleinen Kammer in Hausform. Architektonisch entspricht dieser an drei Seiten in den Fels eingelassene Bau oikos-Kammergräbern, wobei das unmittelbarste Vergleichsbeispiel das Kenotaph für den Stadtgründer Battos auf der Agora von Kyrene ist ${ }^{5}$. Nach Aufbringen der Erdschicht war allerdings in Kyrene wie in Poseidonia nur mehr ein Hügel sichtbar.

Sechs Meter östlich der unterirdischen Grabkammer und an dieser orientiert wurden drei Steinblöcke in den Boden eingelassen. Diese gehörten zum Unterbau einer vor dem Hügel gelegenen Einrichtung, am ehesten zu einen Altar ${ }^{6}$.

3 Zur städtebaulichen Entwicklung von Poseidonia vor allem die Beiträge von E. Greco, La città e il territorio: problemi di storia topografica, S. 471ff, und D. THeodoRESCU, Éléments d'urbanisme et de topographie. État actuel et perspectives, S. 501ff., beide publiziert in Poseidonia-Paestum, Atti del XXVII. Convegno di Studi sulla Magna Grecia, Tarent, 1987 [1988].

4 Theodorescu, op. cit. (n. 1), S. 64f. Die östliche Begrenzung des Areals läßt sich aufgrund der durch das Grabungsgelände verlaufenden modernen Straße nicht mehr feststellen.

5 Ausführliche Beschreibung und Diskussion des Befundes sowie ein Vergleich mit dem Kenotaph des Battos in Kyrene durch Theodorescu - Greco, op. cit. (n. 1), 28-33 u. 74f. Zum archäologischen Befund des Monuments für Battos, S. STuccHI, L'agorà di Cirene I. I lati nord ed est della platea inferiore, Rom, 1965 (Monografie di Arch. Libica, VII), S. 111-114; zur politisch-sozialen Bedeutung des Monuments, M. MüLLER, Grabmal und Politik in der Archaik, in W. Hoepfner, G. Zimmer (hrsg.), Die griechische Polis. Arcbitektur und Politik, Tübingen, 1993, S. 63-66.

6 Kron, art. cit. (n. 1), S. 118f. mit Abb. 1; Theodorescu - Greco, op. cit. (n. 1), s. $32 \mathrm{f}$. 76, Fig. 14, 18/B, C. 
Innerhalb der Kammer ${ }^{7}$ waren längs der Nord- und Südwand je vier Bronzegefäße, insgesamt sechs Hydrien und zwei Amphoren, aufgestellt. Diese Gefäße waren mit einer zähflüssigen Masse, die allgemein als Honig gedeutet wird, gefüllt. In der Nordostecke stand eine attisch-schwarzfigurige Bauchamphora, die auf der Schauseite die Apotheose des Herakles zeigt. In der Mitte des Raums befand sich ein aus zwei Kalksteinblöcken gebildetes, niederes Podest (L. $0,92 \mathrm{~m}, \mathrm{Br} .0,68 \mathrm{~m}, \mathrm{H} .0,54 \mathrm{~m}$ ) auf dem fünf Eisenstangen und Reste von Holz, Gewebe sowie eines Metallbandnetzes gefunden wurden.

Die innerhalb der späteren Ummauerung des Monuments gefundenen Gefäßragmente des 6. Jh. v. Chr. sowie zahlreiche Tierknochenfunde sind mit jüngerem, zum Teil erst aus römischer Zeit stammendem Material vermischt ${ }^{8}$. Es ist also nicht zu entscheiden, wann diese an ihren Fundort verbracht wurden und ob alle oder zumindest ein Teil dieser Funde mit dem Kult innerhalb der Grabkammer zu verbinden sind. Gemeinsam mit den schon erwähnten Steinblöcken östlich des Oikos bzw. des diesen deckenden Hügels machen die Tierknochenfunde regelmäßig durchgeführte Schlachtopfer vor dem Monument aber zumindest wahrscheinlich.

\section{Der Zeitpunkt der Errichtung des Hypogäums}

Das Hypogäum wurde aufgrund der innerhalb der Kammer gemachten Funde im letzten Viertel des 6. Jh. v. Chr. errichtet ${ }^{9}$. Diese Baumaßnahme stand also nicht in Verbindung mit der grundsätzlichen Konzeption einer öffentlichen Freifläche im Zentrum des Siedlungsgebietes im frühen 6. Jh. v. Chr., sondern erfolgte zeitgleich mit dem Baubeginn des ersten monumentalen Athenatempels und eines weiteren Kultbaus, der am Südrand der Agora, unter dem späteren Komplex "Curia-Macellum", errichtet wurde ${ }^{10}$. Dieser Tempel ist von besonderem Interesse, da er in seiner Orientierung von

7 Kron, art. cit. (n. 1); S. 124, 121 Abb. 2 (Zeichnung des Kammerinnenraums), 127 Abb. 6-8 (Eisenstangen, Reste eines Metallbandnetzes und von Gewebe).

8 Die Keramikfunde wurden von SESTIERI, art. cit. (n. 1), S. 54f, 63f, genannt und von Theodorescu - Greco, op. cit. (n. 1), S. 139-145, gemeinsam mit den im Zuge der Nachgrabungen gefundenen jüngeren Objekten publiziert. Zu den 'Tierknochenfunden, P.C. Sestieri, Paestum, in Itinerari dei Musei, Gallerie e Monumenti d'Italia, No. 84, $1967^{10}$, S. 27; KRON, art. cit. (n. 1), S. 121f.

9 Diese Datierung basiert auf der stilistischen Entwicklung der innerhalb der Grabkammer gefundenen Gefäße, vor allem der attisch-schwarzfigurigen Amphore mit der Darstellung der Apotheose des Herakles; Sestieni, art. cit. (n. 1), S. 63 Abb. 23.24, 64; Kron, op. cit. (n. 1), S. 124 m. Anm. 20 u. 127 m. Anm. 37. Die Funde aus der die Kammer deckenden Erdschicht stammen dagegen aus unterschiedlichen Epochen und können daher, wie Greco, op. cit. (n. 1), bes. S. 74ff, gezeigt hat, zur Festellung des Zeitpunktes der Errichtung des Monuments nicht herangezogen werden.

10 Theodorescu, op. cit. (n. 1), S. 32 mit Anm. 20. 
den Sakralbauten im nördlichen wie im südlichen Stadtheiligtum abweicht ${ }^{11}$. Er orientierte sich dabei an jener Geländewelle, die die Agora im Süden begrenzte, und zwar in einer Achse, an der sich später auch die Bauten auf dem römischen Forum ausrichteten ${ }^{12}$. Städtebaulich hatte dieser Tempel dieselbe Funktion wie das Hypogäum: eine Definition der Grenze des öffentlich nutzbaren Agorabereichs.

\section{Zwischenergebnis}

Aufgrund dieser gesicherten Befunde lassen sich die folgenden Aussagen über die Art des Monuments machen:

1) Es lag auf der Agora von Poseidonia. Das Monument hatte also primär politischen Charakter.

2) Der Bau der unterirdischen Kammer, ihre Einrichtung sowie die Deckung der Anlage mit einem Erdhügel erfolgten in einem Zug.. Die unterirdische Lage der Kammer, ihre architektonische Form und die in der Kammer deponierten Gegenstände entsprechen gleichzeitig einem für Heroen und chthonische Gottheiten üblichen Ritus (mit Honig gefüllte Gefäße, unterirdische Lage der Kammer, formale Übereinstimmung mit Kammergräbern) wie auch einer für olympische Götter übliche Verehrung (Spieße für ein Brandopfer ${ }^{13}$ ).

3) Die östlich des Hügels gefundenen Steinblöcke zeigen, daß es vor dem neu angelegten Hügel eine weitere bauliche Einrichtung, am ehesten einen Altar, gab. Regelmäßige Kulthandlungen an dieser Stelle sind wahrscheinlich.

4) Der Zeitpunkt der Errichtung der Anlage im letzten Viertel des 6. Jh. erfolgte zeitgleich mit der Einrichtung eines die südliche Grenze des Agoragebietes definierenden Tempels. Wie dieser markierte das Monument im Westen den Übergang des öffentlichen Agorabereichs zum anschließenden Wohngebiet. Dies bezeugt indirekt eine rechtliche Definition dieser öffentlich nutzbaren Fläche in einer Weise, wie sie etwa in Athen im späten 6. Jh. v. Chr, durch die Setzung von Grenzsteinen der Agora durchgeführt wurde ${ }^{14}$.

11 Zu diesem Bau ausführlich D. Theodorescu, in D. Theodorescu, E. Greco, Poseidonia-Paestum I. La "Curia", Rom, 1980 (Coll. de l'École française de Rome, 42), S. 25-27, Fig. 45.

$12 \mathrm{Zu}$ dieser Geländewelle, die das Agoragebiet im Süden begrenzt, und zu den später an dieser orientierten Bauten, Theodorescu, op. cit. (n. 1), S. 64.

13 Als solche wurden diese von KroN, art. cit. (n. 1), S. 131-144, identifiziert.

14 G.V. Lalonde (mit M.K. Langdon u. M.B. Walbank), The Athenian Agora XIX: Inscriptions. Horoi. Poletai Records. Leases of Public Lands, Princeton, 1991, H 25-29; zur grundsätzlichen Bedeutung dieser und vergleichbarer Grenzsteine öffentlicher Flächen, J. OBER, Greek Horoi: Artifactual Texts and the Contingency of Meaning, in D.B. SMALL (hrsg.), Methods in the Mediterranean, Leiden, 1995, S. 91-123; M. RauscH, Isonomia in Atben. Veränderungen des öffentlichen Lebens in Atben zwischen dem Sturz der Tyrannis und der zweiten Perserabwebr, Frankfurt, 1999, S. 11-15, $311 \mathrm{ff.}$ 


\section{Die Zeremonien zur Verehrung der Tritopatores in Selinunt}

In einem um die Mitte des 5. Jh. publizierten Sakralgesetz aus Selinunt, das sich mit der Reinigung blutschuldiger Personen befaßt, werden detailliert Kulthandlungen zu Ehren der Tritopatores, der kollektiv verehrten Vorfahren der selinuntiner Bürgerschaft, beschrieben (Kolumne A, Z. 9-17) ${ }^{15}$ :

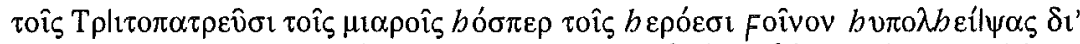

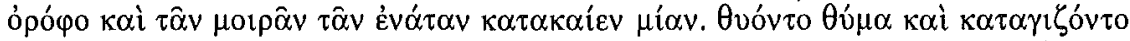

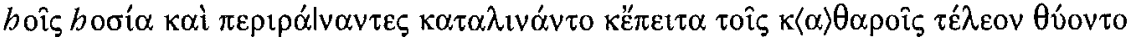

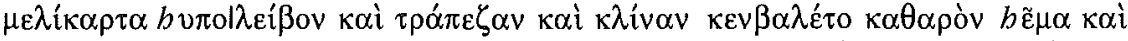

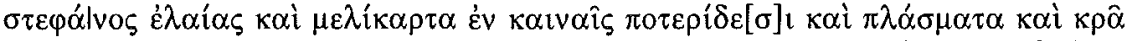

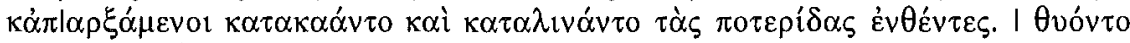

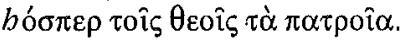

Die Rituale lassen sich folgendermaßen zusammenfassen ${ }^{16}$ :

1) Die Tritopatores werden in zweifacher Form verehrt: als "befleckte"

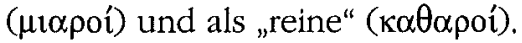

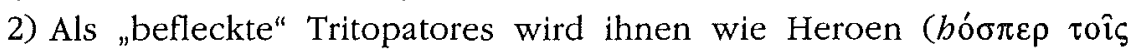

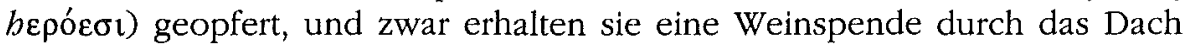
(ihrer unter der Erde gelegenen Kultstätte) sowie ein Brandopfer.

3) Nach vollzogener Reinigung wird den „reinen“ Tritopatores ein Schaf geopfert. Danach wird ihnen eine Trankspende in Form eines Honiggetränks $\left(\mu \varepsilon \lambda i_{\kappa} \alpha \rho \tau \alpha\right)$ dargebracht. Schließlich wird ihnen (bzw. ihren Standbildern) ein Fest vor ihrer Kultstätte bereitet, für das sie eigens mit gereinigten Gewändern bekleidet und mit Olivenzweigen bekränzt werden. Das dabei gereichte Opfermahl wurde auf einem Tisch angerichtet und bestand erneut aus einem Honiggetränk, das in neuen Gefäßen serviert werden sollte, aus Opferkuchen und Fleisch.

4) Die vollzogenen Rituale entsprechen den von den Vorfahren überlieferten und allen Selinuntinern gemeinsamen Sitten $(\pi \alpha \tau \rho \circ \hat{\imath} \alpha)$, die sie als Angehörige einer politischen Gemeinschaft von Bürgern anderer Städte unterschieden ${ }^{17}$. Die im Rahmen des Kultes geehrten „heiligen Gegenstände“,

15 M.H. Jameson, D.R. JoRdan, R.D. Kotansky, A lex sacra from Selinous, Durham, 1993 (GRBS Monograph, 11); Text mit Kommentar auch bei R. ArenA, Iscrizioni grecbe arcaicbe di Sicilia e Magna Grecia. Iscrizioni della Sicilia, I. Iscrizioni di Megara Iblea e Selinunte, Pisa $1996^{2}$, S. $104-115$.

16 JAMESON - JORDAN - KOTANSKY, op. cit. (n. 15), S, 29-37 (Textkommentar) u. 63-73 (Diskussion des Rituals); K. Clinton, Review Article. A new Lex Sacra from Selinus: kindly Zeuses, Eumenides, impure and pure Tritopatores, and Elasteros, in CPh, 91 (1996), S. $170-172$.

17 Dies wird in der zweiten Kolumne (B) der Tafel aus Selinunt deutlich, wo in Zeile

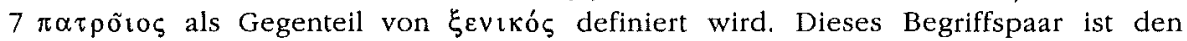

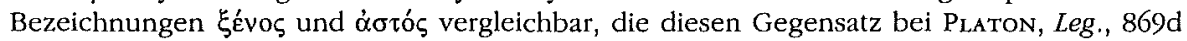


wohl die Kultbilder der Tritopatores, sind öffentlicher Besitz (A, Z. 18: br $\alpha \dot{\alpha}$ $\tau \grave{\alpha} \delta \alpha \mu o ́ \sigma(\alpha)^{18}$.

Die in diesem Sakralgesetz aus Selinunt beschriebenen Zeremonien im Heiligtum der Tritopatores entsprechen in mehreren Punkten jenem Ritual, das anläßlich der Einrichtung der unterirdischen Kammer auf der Agora von Poseidonia vollzogen wurde.

Ein Vergleich der beiden Heiligtümer muß allerdings den grundsätzlichen Unterschied der Evidenz berücksichtigen. Während in Selinunt immer wieder und zu einem ganz bestimmten Zweck (nämlich der rituellen Reinigung von Blutschuld) durchzuführende Zeremonien beschrieben sind, die auch Rückschlüsse auf das Aussehen des Kultortes der Tritopatores erlauben, ist in Poseidonia nur ein einmaliger Stiftungsakt und die architektonische Form eines öffentlichen Monuments auf der Agora faßbar.

\section{Die Übereinstimmungen zwischen der Kultstätte der Trito- patores in Selinunt und dem Hypogäum von Poseidonia}

Ebenso wie das Heiligtum der Tritopatores in Selinunt lag auch die Kultstätte auf der Agora von Poseidonia unterirdisch. Eine Öffnung im Dach der Kultkammer, wie sie für Selinunt bezeugt ist, gab es jedoch nicht.

Ebenso wie die Tritopatores sowohl in der für Heroen wie auch Götter üblichen Art geehrt werden, ist eine solche parallele Durchführung von heroischen und göttlichen Opfern ${ }^{19}$ auch für die Stiftungszeremonie des Hypogäums von Poseidonia nachweisbar. Das den Tritopatores in Selinunt gespendete Honiggetränk entspricht dabei der in den Bronzegefäßen im Hypogäum von Poseidonia deponierten honigartigen Masse, das im

aber auch schon in archaischen Gesetzestexten ausdrücken; zu den letztgenannten epigraphischen Zeugnissen, H. van EfFenterre, F. Ruzé (hrsg.), Nomima. Recueil d'inscriptions politiques et juridiques de l'archaisme grec, I, Rom, 1994, S. 29ff. Vgl. aber auch JAMESON - JORDAN - KOTANSKY, op. cit. (n. 15), S. 36f. u. 44, mit einer abweichenden

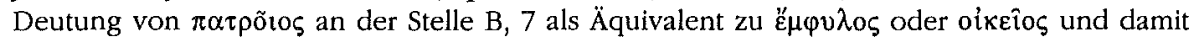
als Bezeichnung der Zugehörigkeit zu einer Sippe.

18 Diese Definition findet sich im abschließenden Teil der Inschrift (A, Z. 18-24), in welchem die Häufigkeit und die rechtliche Grundlage zur Durchführung der beschriebenen Rituale sowie Rechte und Pflichten der Opfergemeinschaft und ihres Priesters bestimmt werden. Zum Verhältnis zwischen Staat, Opfergemeinschaft und Priester in diesem Gesetz nun ausführlich, M. RAusch, Damos, gruppi ed individui in una lex sacra di Selinunte, in Minima Epigrapbica et Papyrologica, III (2000), p. 39-52.

19 HDT, II, 44, 5 bezeugt eine derartige Verehrung als olympische Gottheit, zugleich aber auch als Heros für Herakles; vgl. auch Paus., II, 10, 1 bezüglich des Kultes des Herakles in Sikyon sowie II, 11, 7 zum Kult des Alexanor in Titane; zur Praxis von Heroenkulten allgemein A.D. Nock, The Cult of Heroes, in Essays on Religion in the Ancient World (hrsg. Z. STEWART), Harvard, 1972, S. 576 [Nachdruck von HTbR, 37 (1944), S. 141-174]; zu den beiden unterschiedlichen Arten chthonischer und olympischer Verehrung zuletzt S. Scullion, Olympian and Chtbonian, in CSCA, 13 (1994), S. 75-119 [non vidi]. 
Kultgesetz aus Selinunt genannte Weinopfer der im Hypogäum deponierten Amphora. Die nach olympischem Brauch dargebrachten Brandopfer in Selinunt sind im Hypogäum von Poseidonia durch die Bratspieße auf dem Opferaltar in der Kultkammer symbolisiert.

Das Arrangement der Bratspieße, Holz- und Gewebereste auf diesem Opferaltar im Hypogäum von Poseidonia kann mit jenem im Sakralgesetz aus Selinunt beschriebenen Kultmahl verbunden werden, bei dem (hölzernen) Kultstatuen der Tritopatores „neue Gewänder" ( $\alpha \beta \theta \alpha \rho o ̀ v ~ b \tilde{E} \mu \alpha)$ angelegt und auf einem Opfertisch Wein, Fleisch und Kuchen serviert wurden ${ }^{20}$.

Der öffentliche Charakter des Kultes der Tritopatores in Selinunt ist durch

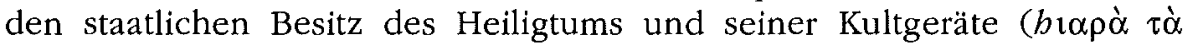
$\delta \alpha \mu o ́ \sigma(\alpha)$ und durch die rechtliche Befugnis der Bürgergemeinschaft, über diese zu verfügen, bezeugt. Dies entspricht dem Akt der Errichtung des Hypogäums auf der im öffentlichen Besitz befindlichen Agora von Poseidonia.

Darüber hinaus ist die Lage des Hypogäums unmittelbar an der zwischen der Porta Aurea im Norden und der Porta Giustizia im Süden verlaufenden Hauptachse der griechischen Stadt ${ }^{21}$ und gleichzeitig am Zugang zur Agora vom westlichen Wohngebiet aus mit der Lage der Heiligtümer der Tritopatores in Athen und auf Delos vergleichbar, wo sich diese an der Kreuzung wichtiger Straßen und unmittelbar vor dem Zugang zur Stadt befanden ${ }^{22}$. Gleichzeitig ist damit eine Verbindung zum Heroon des Gründers Battos in Kyrene gegeben, das auch am (in diesem Fall östlichen) Rand der Agora lag ${ }^{23}$.

\section{Überlegungen zu einem Heiligtum der Tritopatores auf der Agora von Poseidonia und den Umständen seiner Errichtung}

Aufgrund dieser Übereinstimmungen ist eine Deutung des Hypogäums von Poseidonia als Heiligtum der Tritopatores der poseidoniatischen Bürgergemeinschaft eine plausible Hypothese, deren Konsequenzen nun kurz diskutiert werden sollen.

Der in Troizen sicher bezeugte Kult der Tritopatores kann seine Übernahme nach Poseidonia erklären, da Angehörige des ursprünglich aus Troizen stammenden Teils der sybaritidischen Bürgerschaft an der Gründung von

\footnotetext{
20 JAMESON - JORDAN - KOTANSKY, op. cit. (n. 15), S. 67ff.

21 Theodonescu, op. cit. (n. 1), S. 19 m. Anm. 22: „Il faut toutefois remarquer que, si une rue avait été tracée à un moment donné en çorrespondance avec cet axe idéal, elle aurait traversé, à la limite, le sacello ou, du moins son enceinte, le situant ainsi du côté opposé à l'Athenaion."

22 I. MALxin, Religion and Colonization in Ancient Greece, Leiden, 1987, S. 211f.

23 Ibid., bes. 212. Die Lage des Grabes des Battos am Rand der Agora von Kyrene

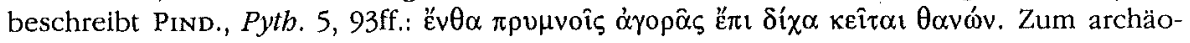
logischen Befund, supra n. 5.
} 
Poseidonia beteiligt waren ${ }^{24}$. Die Wahl der Agora als Ort des Kultplatzes dieser allen Poseidoniaten gemeinsamen Vorfahren ist in Analogie zur Stellung ihres Kultes in Kyrene zu sehen ${ }^{25}$. Dort wurde das Heiligtum der Tritopatores in einem im 4. Jh. publizierten Sakralgesetz seiner kultischen Bedeutung nach mit dem Grab des heroisch verehrten Gründer Battos und jenem eines gewissen Onymastos aus Delphi gleichgesetzt ${ }^{26}$. Diese beiden Einzelpersonen und die Tritopatores wurden gemeinsam für die Gründung von Kyrene verantwortlich gemacht und sollten das Wohlergehen dieser Stadt auch künftig sichern ${ }^{27}$. Eine solche Aufgabe hätten in Poseidonia die Tritopatores erfüllt $^{28}$, die dafür ein Heiligtum am Rand der Agora und zugleich am Zugang $\mathrm{zu}$ derselben erhalten hätten ${ }^{29}$ und mit ähnlichen Zeremonien verehrt worden wären wie die heroisierten Gründer in anderen Städten ${ }^{30}$.

Das Ersetzen eines individuellen Gründers durch das Kollektiv der Vorfahren aller Bürger war notwendig, wenn nicht ein einzelner Oikist als Identifikationsfigur aller Angehöriger der politischen Gemeinschaft zur Verfügung stand. Ein solches Fehlen ist in Poseidonia aus dem Gegensatz zwischen den sybaritidischen Siedlern achäischer und troizenischer Herkunft zu erklären, die gemeinsam in der neugegründeten Tochterstadt von Sybaris siedelten ${ }^{31}$. In der Mutterstadt Sybaris hielt sich dieser Gegensatz hartnäckig und eskalierte schließlich sogar in einer gewaltsamen Auseinandersetzung, die zur Vertreibung der troizenischen Sybariten führte ${ }^{32}$. Es ist wahrscheinlich, daß

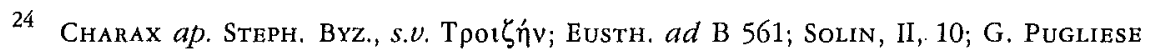
Carratelli, Per la storia di Poseidonia, in Poseidonia-Paestum, Atti del XXVII. Convegno di Studi sulla Magna Grecia 1987, Tarent, 1988, S. 14ff.

25 SEG, IX, 72; XX, 717; F. SokolowsKI, Lois sacrées des cités grecques, Supplément, Paris, 1962, Nr. 115 A 21-25; R. PARKer, Miasma. Pollution and purification in early Greek religion, Oxford, 1983, S. 332-351 (engl. Üs, und ausführliche Diskussion); JAMEsoN JoRdAN - KotANSKY, op. cit. (n. 15), s, 110 f.

26 An diesen drei Kultorten konnte eine „gereinigte“ Person keine öбı empfangen. Die Bedeutung beider Begriffe ist umstritten; siehe die Diskussion bei PARKER, op. cit. (n. 25), S. 336f.; W. LESCHHORN, Grïnder der Stadt. Studien zu einem politiscb-religiösen Phänomen, Stuttgart, 1984 (Palingenesia, XX), S. 67f.; Mal.kin, op. cit. (n. 22), S. 206ff., bes. 210-212.

27 JAMESON - JORDAN - KOTANSKY, op. cit. (n. 15), S. 110f.: „The group as a whole represents the figures who established the settlement and set it on the right path founding hero of the settlement, the community's ancestral spirits, and perhaps an early religious adviser."

28 U. von Wilamowitz-MoellendorfF, Kyrene, Berlin, 1928, S. 9 Anm. 1, deutete in diesem Sinn das spätere, im 5. Jh. auf der Agora von Kyrene angelegte Kenotaph als gemeinsamen Kultort des Battos und der Tritopatores.

29 Supra n. 23.

$30 \mathrm{Zu}$ den besonders von Kallim., Ait. II, fr. 43 beschriebenen Zeremonien zu Ehren eines heroisierten Oikisten ausführlich, LESchHORN, op. cit. (n. 26), S. 98-105

31 Supra n. 24.

32 Aristr., Pol., 1303a 29; Solin, II, 10; Pugliese Carratelli, op. cit. (n. 24), S. 14. 
dieser für die Mutterstadt bezeugte Gegensatz zwischen Bürgern achäischer und troizenischer Abstammung in Poseidonia ebenso eine politische Rolle spielte wie in zahlreichen anderen Kolonialstädten, die von Siedlern unterschiedlicher Herkunft gegründet wurden. Die Einführung eines staatlichen Kultes der Tritopatores konnte die Spannungen zwischen diesen unterschiedlichen Gruppen ausgleichen ${ }^{33}$. Einen staatlichen Charakter dieses Kultes setzt schon die Lage des Hypogäums auf der Agora von Poseidonia voraus, ein solcher entspricht gleichzeitig der staatlichen Verehrung der Tritopatores in Selinunt, in Athen und Kyrene, sowie vielleicht in Troizen ${ }^{34}$.

Die Errichtung des Hypogäums am Westrand der Agora ist zeitlich wie auch bezüglich seiner städtebaulichen Bedeutung in Verbindung mit dem Bau des Tempels an deren Südrand zu sehen. Beide Kultbauten dienten einer Definition jenes Bereichs, der zu einer öffentlichen Nutzung frei gehalten werden sollte. Diese Maßnahme entspricht also der Setzung von Grenzsteinen der Agora, wie sie etwa in Athen am Ende des 6. Jh. v. Chr. erfolgte ${ }^{35}$ und ist damit Teil eines größeren städtebaulichen Konzepts.

In diesem Zusammenhang ist zu erwähnen, daß in Athen bald nach der kleisthenischen Verfassungsreform nicht nur das Areal der Agora, sondern auch jenes des Heiligtums der Tritopatores durch die Setzung neuer Grenzsteine definiert bzw. bestätigt wurden ${ }^{36}$. Ein solcher Verfassungswechsel ist allerdings aufgrund der mangelhaften Kenntnis der poseidoniatischen Stadtgeschichte im späten 6. Jh. v. Chr. nur als Hypothese zu vertreten.

Dies gilt auch für andere mögliche Anlässe zur Errichtung des Hypogäums auf der Agora. Ebenso plausibel ist etwa eine Verbindung mit der Zerstörung von Sybaris im Jahr 510 v. Chr. und dem darauf erfolgten Zuzug eines Teils der Sybaritiden nach Poseidonia. Diese grundsätzlich P. Zancani Montuoro ${ }^{37}$ folgende Deutung stößt jedoch auf das Problem der unsicheren Datierung der Errichtung des Hypogäums (diese ist aufgrund des archäologischen

33 Eine andere, in zahlreichen Kolonialstädten nachweisbare Art der Bewältigung dieses Konflikts war die kultische Verehrung eines Gründers und die gleichzeitige Unterdrückung der Erinnerung an die sonstigen am Gründungsprozeß beteiligten Personen. Dazu ausführlich MaLKin, op. cit. (n. 22), S. 254-260.

34 JAMESON - JORDAN - KOTANSKY, op. cit. (n. 15), s. 107ff., bes. 111. Auf lokaler Ebene ist ein öffentlicher Charakter des Kultes der Tritopatores in den attischen Demen Marathon und Erchia bezeugt. In Marathon opferte der Demarch den Tritopatores jedes Jahr im Namen der Bürger des Demos, IG $\mathrm{II}^{2}$, 1358. Dies entspricht der Sitte in Erchia (SEG, XXI, 541), wobei die das Opfer ausführende Person hier nicht eigens genannt ist.

35 Supra n. 14.

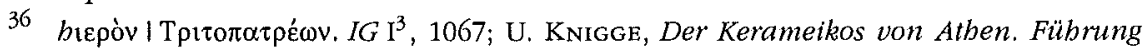
durch Ausgrabungen und Geschicbte, Athen, 1988, S. 104 Abb. 97; JAMESON - JORDAN KotANSKY, op. cit. (n. 15), S. 107; Rausch, art. cit. (n. 14), S. 311ff., bes. 314.

37 P. Zancani Montuoro, La fine di Sibari, in RendLinc, 35, 3-4 (1980), S. 1f; vgl. auch Zancani Montuoro, art. cit. (n. 2). 
Befundes eher vor als nach 510 v. Chr. anzusetzen ${ }^{38}$ ), sowie auf das Fehlen weiterer Belege für eine einschneidende Veränderung des öffentlichen Lebens in Poseidonia am Ende des 6. Jh. v. Chr. ${ }^{39}$. Sie steht darüber hinaus in Widerspruch zur Nachricht Herodots, VI, 21, wonach die Sybaritiden nach der Zerstörung ihrer Stadt in Laos und Skidros Zuflucht gesucht hätten ${ }^{40}$. Die in Selinunt und Kyrene bezeugte Zuständigkeit der Tritopatores für die rituelle Reinigung von Blutschuld macht schließlich auch die Entsühnung der Stadt von einem kollektiv begangenen Unrecht zu einem plausiblen Anlaß für die Errichtung des Heiligtums. Dieser Akt wäre als Reaktion auf einen Orakelspruch zu sehen, der von den Poseidoniaten infolge anhaltenden Unheils (etwa Mißernten oder Seuchen) eingeholt worden wäre ${ }^{41}$. Auch dafür fehlen jedoch eindeutige Belege.

Mario RausCH

Währingerstraße $46 / 1 / 26$

A - 1090 WIEN

38 So schon Sestieri, art. cit. (n. 1), S. 64 und KRon, art. cit. (n. 1), S. 127.

39 Eine solche ist der Wechsel des poseidoniatischen Münzfußes vom phokäischen zum achäischen Standard, der jedoch, wie C.M. KRAAY, Gli stateri a doppio rilievo di Posidonia, in AttiMGrecia, 8 (1967), bes. S. 130ff., gezeigt hat, erst nach $476 \mathrm{v}$. Chr, anzusetzen ist.

$40 \mathrm{Zu}$ diesem und weiteren historischen Einwänden gegen die Theorie von ZaNCANI Montuoro, art. cit. (n. 37), vor allem Greco, op. cit. (n. 1), S. $76 \mathrm{ff}$.

41 In diesem Sinn bereits KRoN, art. cit. (n. 1), S. 147. 